pp 1032-1052. (C) Royal Aeronautical Society 2019.This is an Open Access article, distributed under the terms of the Creative Commons Attribution licence (http://creativecommons.org/licenses/by/4.0/), which permits unrestricted re-use, distribution, and reproduction in any medium, provided the original work is properly cited. doi:10.1017/aer.2019.45

\title{
Effect of scramjet inlet vortices on fuel plume elongation and mixing rate
}

\author{
J.R. Llobet \\ juanrallg@gmail.com \\ R.J. Gollan and I.H. Jahn \\ Centre for Hypersonics \\ School of Mechanical and Mining Engineering \\ University of Queensland, Brisbane \\ Australia
}

\section{ABSTRACT}

Hypersonic air-breathing propulsion can improve cost and flexibility of Low Earth Orbit (LEO) satellite launch missions. However, at the high flight Mach numbers required for access-to-space, performance margins are extremely tight. Techniques to improve mixing efficiency can push this technology forward. However, these are required to produce a minimal increase in losses and heat loads to be viable. The use of inlet-generated vortices in scramjets for mixing enhancement was previously studied. These vortices interact with the injected fuel plume, stretching it and increasing its effective surface for mixing. Moreover, these vortices are intrinsic to the flowfield. Therefore, contrary to other methods, when using inlet vortices mixing is enhanced without producing additional heat loads or losses. This work studies the vortex-injection interaction through numerical RANS simulations. A non-dimensional variable defining the quality of the plume shape for mixing purposes is proposed. This parameter is used to assess the effect of vortex intensity and injector location on fuel plume shape. The results show the ability of inlet vortices to modify fuel plume shape significantly increasing fuel mixing rate with minimal impact on losses.

\subsection{INTRODUCTION}

Hypersonic air-breathing engines, such as scramjets, have the potential to reduce cost and increase flexibility of low earth orbit (LEO) and sun synchronous orbit (SSO) satellite placement missions. By intaking atmospheric air, scramjet engines remove the requirement of carrying oxidiser. Therefore, scramjets have a theoretically higher specific impulse, efficiency, and payload mass fraction than rocket propulsion ${ }^{(1,2)}$. Although scramjet technology has been proven operational through experimental programmes such as the X-43 and X-51 flight demonstrators $^{(3-5)}$, the high Mach numbers required for access to space greatly increase the 
difficulty of thrust production and heat management. Mixing efficiency is one of the key parameters that requires improvement to increase engine performance. Technologies using strong vortices to enhance mixing, such as strut injection and hypermixers, provide substantial improvement in mixing efficiency. Simple struts have been used to successfully increase fuel plume penetration $^{(6,7)}$. Addition of vortex generating shapes to the struts also produces a substantial increase in mixing rate ${ }^{(8,9)}$. As an example, the work by Aravind ${ }^{(8)}$ shows a lobbed strut reduces the distance to $95 \%$ mixing efficiency by about half at the cost of an increase in about $30 \%$ in total pressure over a simple strut at Mach 2.0. Ramps have shown improvement in penetration and mixing by producing vortical structures that interact with the injected fuel. Delta wing ramps of a size similar to the boundary layer showed improved mixing, reducing distance to $95 \%$ mixing efficienty by more than halve, only inducing total pressure losses of about 34\%, albeit at Mach 3.75(10,11) . The mixing improvement generated by these elements is linked to the generation of streamwise vorticity, with the ramps producing higher losses as mixing enhancement is improved ${ }^{(12)}$. These methods have shown very promising improvements in mixing rate. Nonetheless, these studies are focused on lower Mach number ranges. Indeed, for high Mach numbers such as those experienced in a scramjet flight path, between Mach 6 to up to Mach 12, the increase in drag and local heat loads ${ }^{(9,13)}$ they would produce makes them unsuitable for this particular application.

A different approach more suitable to the high Mach numbers experienced in a scramjet flight path, from Mach 6 to up to Mach 12, is to utilize vortices already present in the flowfield. The use of scramjet inlet generated vortices for mixing enhancement takes advantage of features intrinsic to the flowfield. Although the intensity of the vortices naturally generated by scramjet inlets is relatively low compared to those generated by struts or ramps/hypermixers, these are intrinsically present in the flowfield. Therefore, the gains obtained in mixing efficiency by exploiting these vortices comes at zero or negligible additional losses and heat loads. Research focused on the effect of these vortices is scarce. However, Malo-Molina et al. ${ }^{(14)}$ incidentally observed the beneficial effect of these naturally occurring vortices when comparing the performance of two different scramjet inlet shapes. Barth et al. ${ }^{(15)}$ also explored tailoring injector placement to take advantage of regions of intrinsically high vorticity in a scramjet combustor, finding faster mixing in regions with high streamwise vorticity.

A particularly interesting area to exploit vortices for mixing is the scramjet inlet. Inlet injection has been extensively utilized in order to gain available length for mixing purposes ${ }^{(15,16)}$. Achieving high levels of mixing for the portion of the fueling performed at the inlet can reduce combustion delay of the mixture further downstream when it reaches the combustor. Inlet vortices are generated in most non-axysimmetric scramjet inlets. These are particularly characteristic in rectangular inlets, where vortices are formed at the corners through shockwave boundary-layer interactions ${ }^{(17)}$. Rectangular inlets are present in many scramjet designs thanks to their benefits for airframe-integration ${ }^{(18-20)}$.

This work utilises computational fluid dynamics (CFD) to study the effect of streamwise vortices as generated by a scramjet inlet on a porthole fuel plume and its ability to improve fuel mixing rate. A simplified inlet-like geometry is used, consisting of a flat plate and compression fin. Two separate studies are performed. The first one provides an overview of the vortex flowfield (without injection). This allows quantifying the potential of this flowfield to affect the fuel plume shape. Although this implies severe simplifications, it highlights the importance of injector placement within the vortex. Moreover, it provides a simplified method to identify the preferred regions for injector placement. The second study incorporates injection, providing insight and data to quantify the benefit of the 
(a)

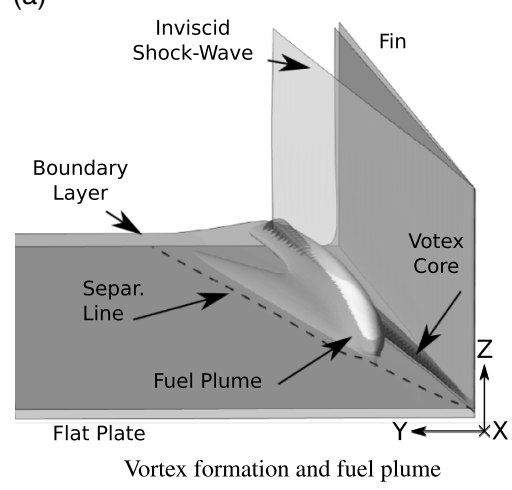

(b)

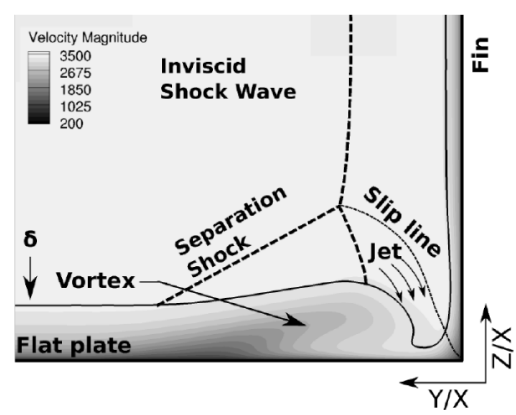

Vortex flowfield structure. $\delta$ and contours from $\alpha_{\text {fin }}=10$ case. Discontinuous lines are adapted from Alvi and Settles ${ }^{(17)}$.

Figure 1. Test geometry and vortex flowfield structure depiction. Adapted from. ${ }^{(24)}$

vortex-injection interaction and also assess the limitations of the first, simplified study. In this case the flat plate incorporates a porthole injector through which hydrogen fuel is injected into the streamwise vortex. The vortex-injection interaction is analysed to characterise the fuel plume shape, the improvement in mixing efficiency produced, and the effect on losses. A number of porthole injector locations, injection-to-freestream injection momentum ratios, and vortex intensities are studied.

\subsection{METHODOLOGY}

\subsection{Vortex generation}

Rectangular scramjet inlets generate streamwise vortices due to shock-shock and shockviscous interactions. To isolate the effect of the streamwise vortex from other features present in the complex flowfield of a scramjet inlet, a simplified canonical geometry is used. This consists of a flat plate and compression wall (see Fig. 1), which produces a single and controlled streamwise vortex ${ }^{(17,21,22)}$. Such a geometry generates vortices representative of those appearing in real screamjet inlet flows ${ }^{(23)}$. The vortex is generated by the interaction of the swept fin shock with the flat plate boundary layer, as depicted in Fig. 1. The swept shock induces the separation of the flat plate boundary layer, which interacts with the incoming freestream to roll up and form the streamwise vortex. Moreover, the streamwise velocity gradient within the flat plate boundary layer produces a gradient in pressure ratio across the fin shock. This generates a secondary flow in the vertical direction moving downwards and outwards, away from the fin, feeding the vortex. This secondary flow is depicted in Fig. 1(b) as "Jet". This flowfield exhibits a quasi-conical behaviour about the fin leading edge ${ }^{(22)}$. This means the flowfield at any axial plane is defined by the flowfield in conical coordinates $\mathrm{Y} / \mathrm{X}$ and $\mathrm{Z} / \mathrm{X}$ as in Fig. 1(b).

\subsection{Flow conditions}

The flow conditions for the current study have been selected to match a MACH 12 scramjet flight condition on a $50 \mathrm{kPa}$ constant dynamic pressure trajectory ${ }^{(20)}$, which has previously been used for ground testing at the University of Queensland. 


\section{Table 1 \\ Free stream and bulk flow near the REST inlet porthole injectors flow conditions ${ }^{(20,21)}$}

$\begin{array}{lcccc}\text { Location } & \text { Mach No. } & \boldsymbol{U} & \boldsymbol{q} & \boldsymbol{H}_{\mathbf{0}} \\ \text { Inlet } & 9.3 & 3523 \mathrm{~ms}^{-1} & 55.0 \mathrm{kPa} & 6.5 \mathrm{MJ} \mathrm{kg}^{-1} \\ \text { Injector region } & 7.3 & 3471 \mathrm{~ms}^{-1} & 161.0 \mathrm{kPa} & 6.5 \mathrm{MJ} \mathrm{kg}^{-1}\end{array}$

Table 2

Vortex characteristics $100 \mathrm{~mm}$ downstream of fin leading edge.

$\begin{array}{ccc}\text { Fin Angle } & \text { Intensity[-] } & \text { Area }\left[\mathbf{1} \times \mathbf{1 0}^{-\mathbf{6}} \mathbf{~ m}^{\mathbf{2}}\right] \\ 5^{\circ} & 0.25 & 27.5 \\ 10^{\circ} & 0.48 & 20.0 \\ 15^{\circ} & 0.65 & 17.5\end{array}$

The REST inlet was first developed by Smart et al. ${ }^{(18,19)}$. This inlet presents a rectangularlike capture area that transitions to an elliptical cross-section for the isolator and combustor. This profits from the the structural and aerodynamic efficiency of a rounded cross-section combustor as well as from the easy airframe integration of a rectangular inlet ${ }^{(18)}$. The corners of the shape transition inlet generate several characteristic vortices that can be exploited to enhance mixing.

Barth et al. ${ }^{(20)}$ present a complete investigation of the flowfield within the REST engine at Mach 12 flight conditions. After accounting for a $6^{\circ}$ angle of attack forebody compression, the freestream conditions in the vicinity of the inlet ramp injectors (Inj. reg.), obtained from full engine simulations conducted by Barth et al. ${ }^{(20)}$, are given in Table 1 . These are the conditions used for the inflow in the current study.

\subsection{Configurations tested}

Two main different typologies are used: the first one, consisting of the flat plate and fin, is used for the vortex flowfield study; the second one is identical but incorporating the injector on the flat plate, and is used to study the fuel plume interaction with the incoming vortex.

\section{Flat plate and fin:}

In the flat plat plus fin geometry, the fin angle $\left(\alpha_{f i n}\right)$ controls vortex intensity. Three different fin angles are used: $5^{\circ}, 10^{\circ}$, and $15^{\circ}$. To assess the intensity and size of the vortices the Q-criterion is used (zones of positive second invariant of $\nabla u)^{(25)}$. The intensity of the vortex is defined as the maximum value of the Q-criterion (occurring at vortex core), and the size is defined as the area enclosing the region with Q-criterion values above $50 \%$ of the vortex intensity. The lowest angle, $\alpha_{f i n}=5^{\circ}$, produces a vortex intensity representative of vortices found in scramjet inlets with low flow non-uniformities. The $\alpha_{f i n}=10^{\circ}$ case produces vortices similar to those found in 2-D inlets with relatively strong corner vortices. The $\alpha_{f i n}=15^{\circ}$ generates a vortex intensity in excess of any corner vortex expected in typical scramjet inlets, and is included for completeness ${ }^{(23)}$. Vortex sizes and intensities for each case are presented in Table 2. For comparison, the REST inlet engine ${ }^{(2)}$, which is designed for developing a highly homogeneous flow, generates a corner vortex with approximate intensity and area of 0.12 and $20 \times 10^{-6} \mathrm{~m}^{2}$ at equivalent conditions. A conventional 2-D engine geometry tested 


\section{Table 3}

Spanwise coordinate, and distance to fin wall for the porthole injectors, $(Y)$ and $\left(Y_{w}\right)$ respectively in Figure 2.

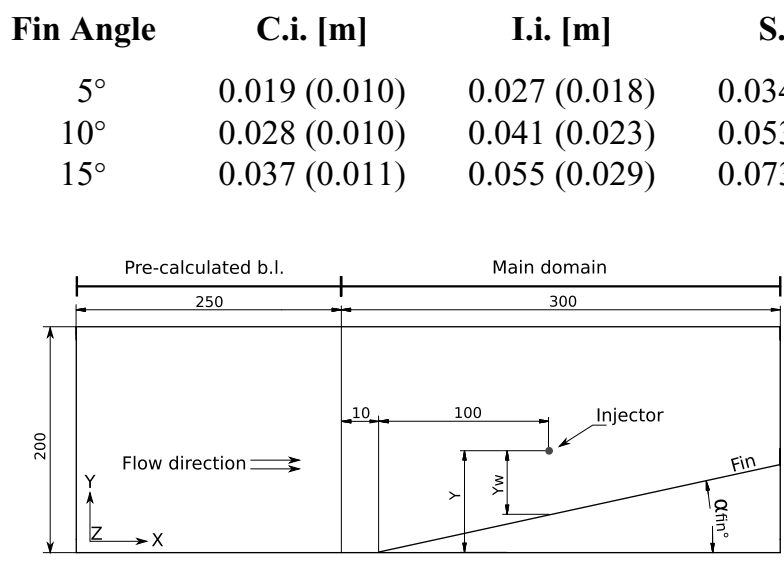

Figure 2. Geometry sketch. Dimensions in $\mathrm{mm} . Y$ and $Y_{w}$ values listed in Table 3.

at the University of Queesland (UQ) ${ }^{(26,27)}$ develops a vortex with approximate intensity and area of 0.5 and $65 \times 10^{-6} \mathrm{~m}^{2}$ at the injector location.

\section{Injection cases:}

Alongside the three different fin angles, the injection-vortex interaction part of the study uses three different injector locations. These locations are based on the location of the vortex separation line and the location of the vortex core, with the following nomenclature:

- Core injector (C.i.): At the normal projection of the vortex core onto the flat plat.

- Separation Injector (S.i.): At the separation line of the swept separation.

- Intermediate Injector (I.i.): At the half-way point between the C.i and S.i.

To complement these cases, the baseline case of injection with no vortex (effectively $\alpha_{f i n}=$ $\left.0^{\circ}\right)$ is used as reference.

The porthole injector has a $1.0 \mathrm{~mm}$ diameter $\left(d_{i n j}\right)$ and is inclined $45^{\circ}$ with respect to the axial direction. As depicted in Fig. 2, the porthole injector is always located $100 \mathrm{~mm}$ downstream of the fin leading edge. The distance to the fin wall (Y. to wall) of the portholes varies for each case, and is measured as the spanwise distance from the centre of the porthole to the fin wall as depicted in Fig. 2. Table 3 shows the coordinates of the porthole injectors relative to the fin leading edge.

In addition, two different injection (inj) to free stream $(\infty)$ momentum ratios $(J=$ $\left.\left(\rho u^{2}\right)_{i n j} /\left(\rho u^{2}\right)_{\infty}\right)$ are tested: $J=1$ and $J=3$. These $J$ values are obtained with the injection conditions summarised in Table 4.

\subsection{Numerical approach}

The CFD solver used for the simulations is US3D, developed at the University of Minnesota $^{(28)}$. Steady state RANS simulations using non-reacting flow and the SST turbulence model with a Schmidt number $(\mathrm{Sc})$ of 0.7 are performed. The typical near-wall cell size is $2 \mu \mathrm{m}$, keeping the $\mathrm{y}+$ values below 1 for the whole domain, except for the first few cells adjacent to the fin leading edge. The Steger-Warming flux vector splitting method is used for 


\section{Table 4 Injection conditions}

$\begin{array}{ccccc}\boldsymbol{J}[-] & \|\boldsymbol{V}\|\left[\mathrm{m} \mathrm{s}^{-\mathbf{1}}\right] & \boldsymbol{\rho}\left[\mathrm{kg} \mathrm{m}^{-\mathbf{3}}\right] & \boldsymbol{T}[\mathbf{K}] & \dot{\boldsymbol{m}}\left[\mathrm{kg} \mathrm{s}^{-\mathbf{1}}\right] \\ 3 & 1207.2 & 0.21 & 249.0 & 0.19 \cdot 10^{-3} \\ 1 & 1207.2 & 0.62 & 249.5 & 0.57 \cdot 10^{-3}\end{array}$

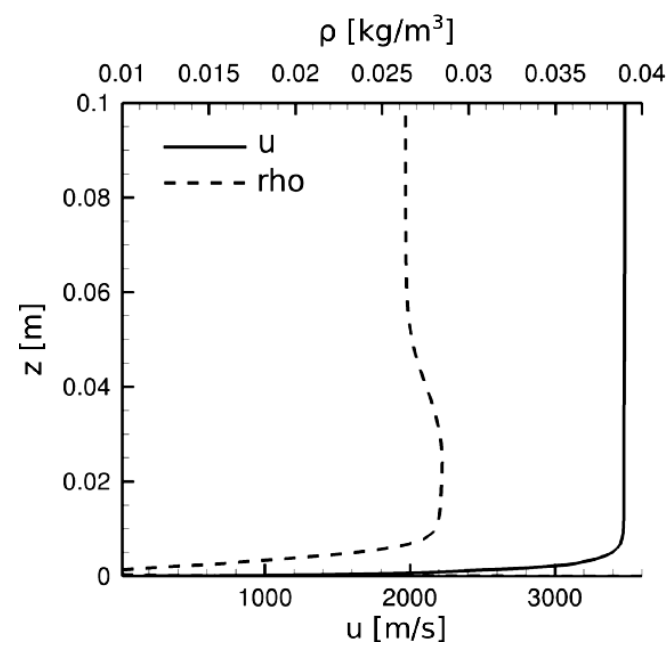

Figure 3. Inflow velocity and density profiles.

the convective fluxes. In regions of strong shocks, the MUSCL scheme with pressure limiter is used. The implicit time integration uses the DPLR method ${ }^{(29)}$. Although CFL value has not been observed to have a significant effect on the final solution, this has been routinely set to 50 towards the end of the simulations.

The numerical domain consists of a $300 \mathrm{~mm}$ long, $200 \mathrm{~mm}$ wide flat plate and a fin $100 \mathrm{~mm}$ high with an infinitely sharp leading edge starting $10 \mathrm{~mm}$ downstream of the inflow and extending to the end of the domain. The domain is sized to avoid any influence of the boundary conditions on the vortex flowfield. Figure 2 shows a sketch of the geometry.

The inflow represents a boundary layer developed over $250 \mathrm{~mm}$ long flat plate positioned upstream of the domain (marked as 'Pre-calculated b.l.' in Fig. 2). This inflow profile was obtained in a separate simulation producing the velocity and density profiles shown in Fig 3 . The fin and flat plate are constant temperature walls set to $300 \mathrm{~K}$, and the back, upper, and opposite side to the fin are set as supersonic outflows.

A grid independence analysis was performed based on the most relevant parameters to be extracted from the simulations, such as mixing efficiency, penetration, and fuel plume shape. The presented results are obtained for the case with fin angle $\alpha_{f i n}=10^{\circ}$, Intermediate Injector (I.i.), and injection to freestream momentum ratio $J=1$. The results for four different levels of mesh refinement are presented in Fig. 4 . The 3.8 million cell mesh was selected for this study. In this mesh, the cell size in the vicinity of the injector is approximately $0.05 \mathrm{~mm}$, expanding up to $1 \mathrm{~mm}$ in the region of uniform flow, far from the vortex-injection interaction. This mesh captures the most relevant aspects of the flowfield satisfactorily. The initial penetration shows no variation between meshes, and the selected mesh only provides a slight underprediction 


\section{Table 5 \\ $\mathrm{GCl}$ for mixing efficiency and maximum penetration}

$\begin{array}{lccc} & \left.\boldsymbol{\eta}_{\text {mix }}\right|_{\mathbf{x}=\mathbf{0 . 0 5}} & \left.\boldsymbol{\eta}_{\text {mix }}\right|_{\mathbf{x}=\mathbf{0 . 0 7 5}} & \boldsymbol{Y} \text { max penetration } \\ G C I_{1-2} & 9.26 & 4.14 & 4.29 \\ G C I_{2-3} & 18.97 & 7.15 & 5.66\end{array}$

(a)

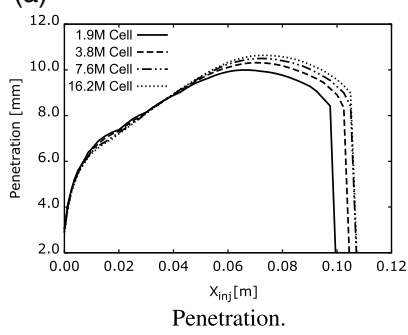

(b)

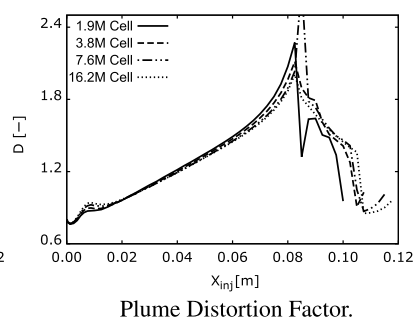

(c)

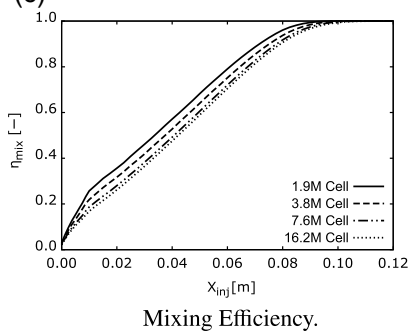

Figure 4. Comparison of mixing parameters for four different levels of mesh refinement.

of the maximum penetration compared to the finest mesh. The plume shape parameter $D$ (defined later on) in Fig. 4(b) shows very similar values to the finest mesh for the region between the injector $\left(X_{i n j}=0.0\right)$ and where the plume splits in two $(\sim X=0.08)$ and the definition of $D$ fails. Similarly, mixing efficiency is retrieved satisfactorily, only showing a small overprediction just downstream of the injector compared to the finest mesh. To further quantify the quality of the 3.8 million cell mesh, the Grid Convergence Index or $\mathrm{GCI}^{(30)}$ for mixing efficiency at two different locations, and for maximum penetration is calculated using the three finest meshes. $G C I_{1-2}$ and $G_{C I_{2-3}}$ are presented in Table 5 based on the coarse mesh (i.e., $G C I_{1-2}$ based on the $7.6 \mathrm{M}$ cells mesh and $G C I_{2-3}$ based on the $3.8 \mathrm{M}$ cells mesh), assuming a 2 nd order level of convergence and using a safety factor of 1.25 . The $G_{C I}-3$ values are the relevant for the $3.8 \mathrm{M}$ cells mesh. The GCI values based on mixing efficiency near injector $\left(\left.\eta_{\text {mix }}\right|_{x=0.05}\right)$ show relatively poor values due to the large concentration gradients in this region. Nonetheless, the GCI value improves further downstream, where the most relevant data is extracted.

A closely related work ${ }^{(31)}$ employing the same methodology on an equivalent geometry and very similar freestream conditions shows good agreement between numerical and experimental heat transfer data. Moreover, Barth et al. ${ }^{(15)}$ used US3D to simulate the full REST engine, which included several injectors into the scramjet internal flow generating a similar interaction to the one described in this paper. The work by Barth presents a more complex flowfield, making simulations more challenging. Despite this, comparison between the numerical and experimental data yielded very satisfactory results. This further increases confidence in the capability of the presented methodology to obtain accurate results.

\subsection{Fuel plume elongation quantification}

Figure 5 shows the interaction of a fuel plume injected within a vortex. This figure clearly depicts the severe change in shape as the fuel plume is convected within the vortex. By interacting with a streamwise vortex, the fuel plume is stretched. This effect can be observed in 


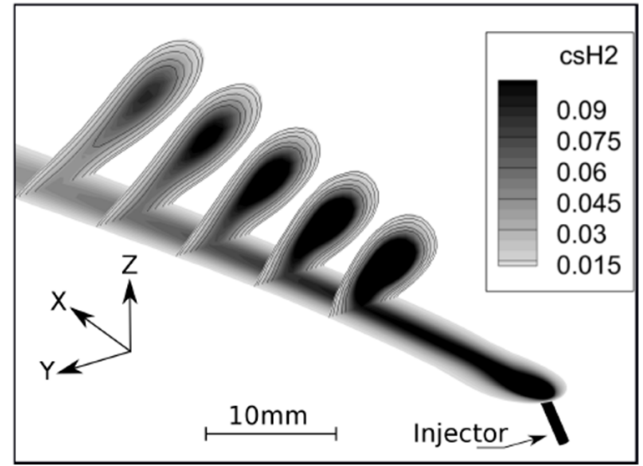

Figure 5. Contours of hydrogen mass fraction depicting fuel plume shape evolution. From separation line injection, $J=1, \alpha_{f i n}=10$ case.

(a)

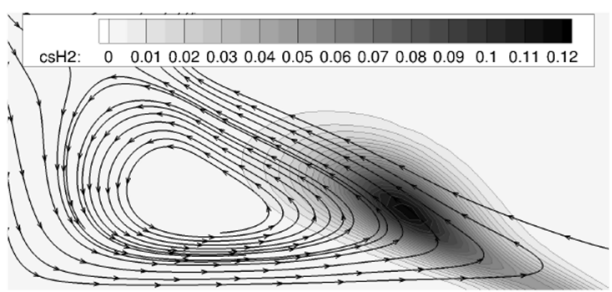

$c_{H 2}$ contours and in plane streamtraces in vortexinjection interaction case. (b)

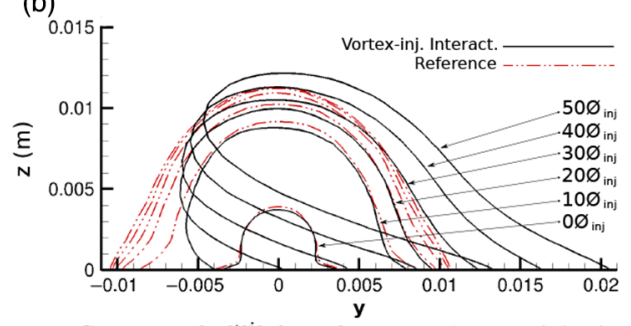

Contours of $c_{\mathrm{H} 2}^{\text {stoic }}$ for reference and vortex-injection interaction cases.

Figure 6. Elongation of the fuel plume ${ }^{(21)}$. As per description in section 2.3, reference case is: $J=3$, $\alpha_{f i n}=0^{\circ}$; vortex-injection interaction case is: Separation injection, $J=3, \alpha_{f i n}=10^{\circ}$.

Fig. 6(a), which depicts an axial slice with in-plane streamlines and contours of fuel mass fraction $\left(\mathrm{Cs}_{\mathrm{H}_{2}}\right)$ for a porthole injection case with vortex-injection interaction. The region of the plume adjacent to the flat plate is subject to an intense crosswise flow, as can be seen in Fig. 6(a). Therefore, this region is strongly convected in the spanwise direction. However, the effect of the vortex on the upper region of the plume is much smaller, and this region follows the velocity of the flow processed by the shock. This velocity gradient causes a shear effect on the plume, elongating it in the spanwise direction. Figure 6(b) shows the evolution of the plume shape at different distances from the injector for cases with and without vortex interaction, clearly showing the stretching caused by the vortex. This elongation results in an increased effective area for mass transfer between the high and low mass fraction regions, hence, increasing mixing rate. A similar mechanism of mixing enhancement was observed in the work by Peterson et al. ${ }^{(32)}$.

To evaluate the suitability of the plume shape for mixing purposes, a dimensionless parameter is proposed. This parameter is based on the ratio of lenth $(L)$ of the stoichiometric mass fraction iso-line length to area $(A)$ of the plume on an axial plane. Higher values of $L / A$ indicate higher effective surface for mixing for a given plume cross-sectional area. As perimeter grows linearly and area grows quadratically, the same plume shape provides different $L / A$ values depending on its characteristic size. In order to avoid this dependency, the $L / A$ value is normalised by the perimeter to area ratio of a circle of the same area $(L / A)_{c}$. This is 
presented in Equation 1. Henceforth, this non-dimensional parameter representative of plume shape will be referred to as $D$.

$$
D=\frac{\frac{L}{A}}{\left(\frac{L}{A}\right)_{c}}=\frac{\frac{L}{A}}{\sqrt{\frac{4 \pi}{A}}}=\frac{L}{\sqrt{4 \pi A}}
$$

The velocity field varies substantially within the vortex region from the vortex core to the separation line, as visible in Figures 1(b) and 6(a). For this reason, injector location plays a major role in the vortex-injection interaction and the degree of fuel plume elongation produced.

\subsection{VORTEX FLOWFIELD PLUME ELONGATION POTENTIAL}

This section contains the first part of the study, analyzing the vortex flowfield and quantifying its ability to produce fuel plume stretching. This is performed without fuel injection, and is meant to provide insight in the vortex flowfield and indentify the preferred location to place the injector.

To estimate the elongation potential in different regions of the vortex, the flowfield generated by the test geometry with a fin angle of $\alpha_{f i n}=10^{\circ}$ is analysed prior to injection. The potential effect of the flowfield on plume shape is studied evaluating the initial and final shape of a streamtube. For this, a number of circles of zero mass are placed on a plane normal to the axial direction, as presented in Fig. 7(a). These are equivalent to a ring of zero-mass particles, which are convected with the flow. Following the trajectories of each particle, the final shape is obtained. To simplify the calculation of the trajectories, the conical nature of the flowfield is exploited. Tracking of each particle is achieved by an iterative two-step process. First, each particle is transported in the direction of the local flow velocity. Second, the new velocity is obtained by re-mapping the new particle location onto the original plane in polar coordinates. Each particle is "tracked" in this fashion until they cross the selected end plane, where the $D$ factor for the new shape is calculated.

\subsection{Results}

The effect of the flowfield on the final shapes is presented in Fig. 7(a), where the streamtubes are initially placed in the $\alpha_{f i n}=10^{\circ}$ flowfield at an axial plane $100 \mathrm{~mm}$ downstream of the fin leading edge. This is the location of the injector in the second part of the study, which in the injector reference system is $x_{i n j}=0$. The streamtube is convected for $40 \mathrm{~mm}$ up to $x_{i n j}=40$.

By tracking multiple streamtubes, the elongation potential for different spanwise positions is obtained. In Fig. 7, the effect of circle diameter $\left(\phi_{c}\right)$ is explored while keeping the minimum distance between the circles and the flat plate $\left(Y_{\min }\right)$ at $0.5 \mathrm{~mm}$. In Fig. 8 the diameters of the circles are kept constant $\left(\phi_{c}=10 \mathrm{~mm}\right)$, while varying the distance between circle centres $\left(Y_{F . P .}\right)$ and flat plate.

Figures 7(b) to 7(d) present the non-dimensinalized perimeter to area ratio $D$ of the final shapes as a function of the starting location of the circle centres in conical coordinates $Y / X$ for three different fin angles. On the plots, the location of the vortex core $(C)$, separation line (S), as well as the Intermediate location between these (I) are also indicated, referencing the location of the injectors used in the second part of this study. These figures shows the importance of vortex intensity and injector location on the potential for fuel plume elongation. 
(a)
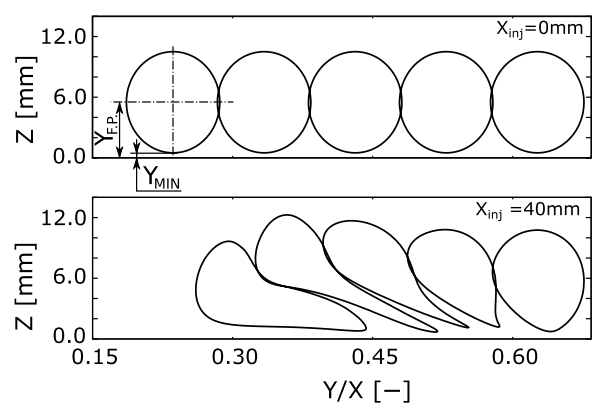

$10 \mathrm{~mm}$ diameter circles at the $X_{i n j}=0 \mathrm{~mm}$ and $X_{i n j}=$ $40 \mathrm{~mm}$ planes for the $\alpha_{\text {fin }}=10^{\circ}$ flowfield.

(c)

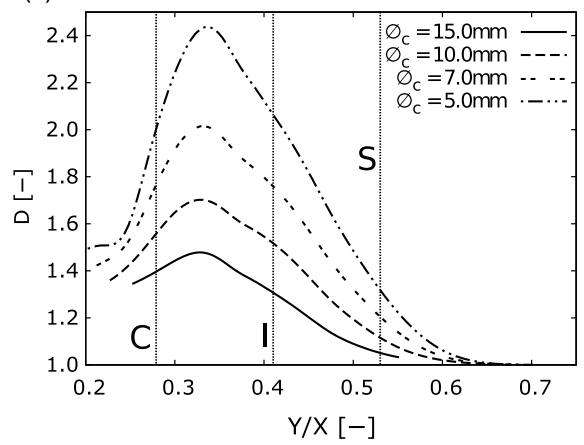

$D$ factor at $X_{i n j}=40 \mathrm{~mm}, \alpha_{\text {fin }}=10^{\circ}$. (b)

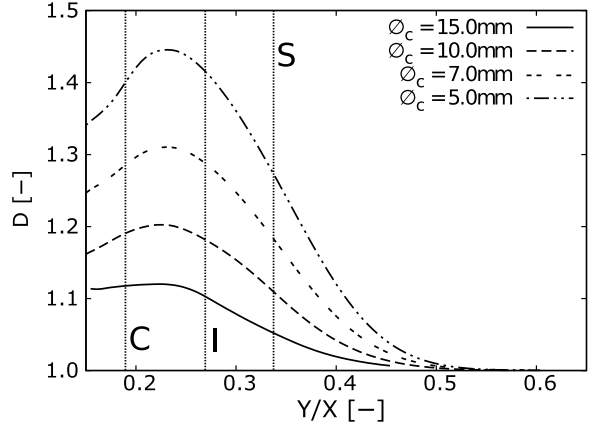

$D$ factor at $X_{i n j}=40 \mathrm{~mm}, \alpha_{\text {fin }}=5^{\circ}$.

(d)

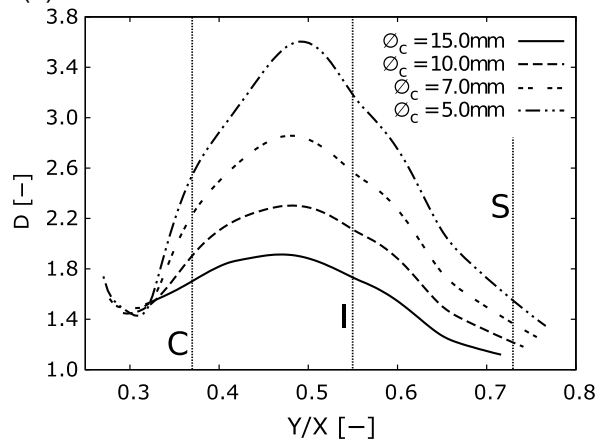

$D$ factor at $X_{i n j}=40 \mathrm{~mm}, \alpha_{\text {fin }}=15^{\circ}$.

Figure 7. Zero-mass cirles elongation. Minimum distance to flat plate is $0.5 \mathrm{~mm}$.

(a)

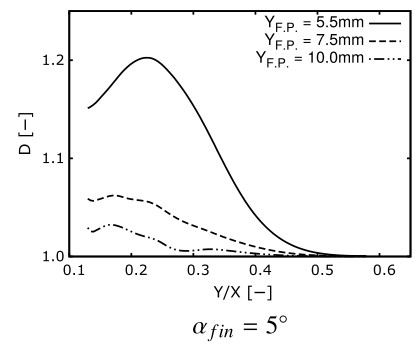

(b)

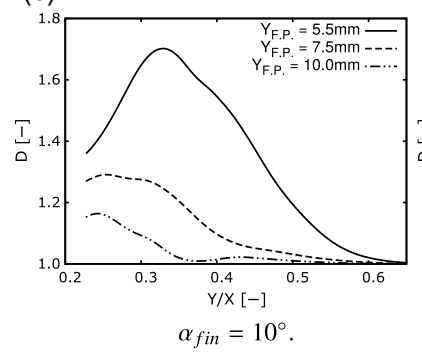

(c)

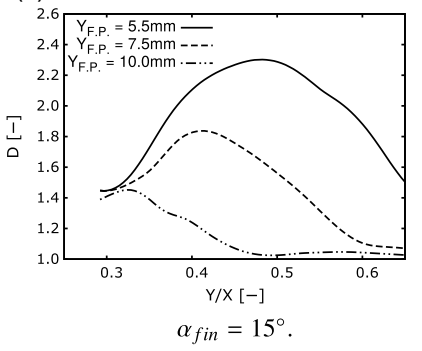

Figure 8. $D$ factor after $40 \mathrm{~mm} . \varnothing_{c}=10 \mathrm{~mm}$, centres at different distances from the plate $\left(Y_{F . P .}\right)$.

The $\alpha_{f i n}=15^{\circ}$ case induces a maximum $D$ value 1.35 times larger than the $\alpha_{f i n}=10^{\circ}$ case, and 1.9 times larger than the $\alpha_{f i n}=5^{\circ}$ case. The size of the circle also plays an important effect. This size can be regarded as the fuel plume size and penetration, controlled by the injector diameter and injection to freestream pressure, with larger diameters corresponding to larger plumes. Smaller circles sit well within the region of highest intensity of the vortex crossflow (Fig. 6(b)), where the velocity gradients are large and produce an intense elongation. 
As the size of the ring increases, the upper region occupies a zone of lower velocity gradient, reducing the overall elongation induced to the circle.

The spanwise location of maximum elongation is constant for the different ring sizes. This location sits at around $Y / X=0.22$ for the $\alpha_{f i n}=5^{\circ}$ case, $Y / X=0.33$ for the $\alpha_{f i n}=10^{\circ}$ case, and $Y / X=0.49$ for the $\alpha_{f i n}=15^{\circ}$ case. These values are consistently at $1 / 4$ of the distance between the vortex core and the separation line.

The effect of circle distance to the flat plate, $Y_{F . P .}$ is presented in Fig. 8. Zero-mass circles with $10 \mathrm{~mm}$ diameter and centres at $5.5 \mathrm{~mm}, 7.5 \mathrm{~mm}$, and $10 \mathrm{~mm}$ from the flat plate evolving for $40 \mathrm{~mm}$ are analysed. Increasing distance to the flat plate can be regarded as equivalent to increasing penetration while keeping plume size relatively constant. The elongation of the circles reduces with increasing distance from the flat plate. Moreover, increasing this distance moves the location of the maximum elongation towards the fin (lower $\mathrm{Y} / \mathrm{X}$ coordinate). As the circles are moved upwards, the interaction with the high velocity gradient crossflow adjacent to the flat plate reduces, decreasing the overall final elongation. Near the fin, the vortex is slightly thicker, as can be seen in Fig. 6(a). This produces certain level of plume elongation to the circles near the fin even at distances relatively far from the flat plate, shifting the location of maximum elongation towards the fin.

This preliminary study indicates that the best injection strategy to enhance mixing rate using inlet vortices involves the use of injectors producing fuel plumes with a size of the order of the boundary layer thickness (thickness of the intense crossflow region within the vortex). This is a suitable strategy for inlet injection as it can generate well mixed regions near the high temperature flow at the walls, initiating the production of radicals, creating regions very suitable to initiate combustion further downstream.

\subsection{VORTEX-INJECTION INTERACTION}

The zero-mass study is a simple and inexpensive method to evaluate the most suitable injection region and strategy to improve plume elongation. To obtain reliable data on the mixing process in the vortex-injection interaction numerical simulations including the injected gas are required. This is addressed in this section, which presents the vortex-injection interaction cases. The effects of this interaction on fuel plume shape and mixing efficiency are quantified. Moreover, the data is used to highlight the limitations of the "zero-mass" study.

\subsection{Plume shape results}

Firstly, the vortex-injection interaction is analysed qualitatively. The evolution of the fuel plume shape for the S.i, $J=1, \alpha_{f i n}=10^{\circ}$ case is depicted in Fig. 5 . The fuel plume is gradually stretched as it moves in the streamwise direction by the action of the vortex velocity field. To observe this effect in more detail, and how injector placement affects this interaction, the spanwise velocity for the $\alpha_{f i n}=10^{\circ}$ case on a plane $10 \mathrm{~mm}$ downstream of the injector (at $X_{i n j}=10 \mathrm{~mm}$ ) is presented in Fig. 9. In this figure, the equivalence ratio lines (Fr) show the location and shape of the fuel plume in the extraction plane. These show that the spanwise velocity gradient in the direction normal to the flat plate is the main driver of the plume elongation.

Depending on injector placement, the fuel plume interacts with a different part of the vortex, which induces different levels of stretching. The Intermediate injector is located adjacent to the high intensity crossflow. This allows the high velocity crossflow to interact with the 
(a)

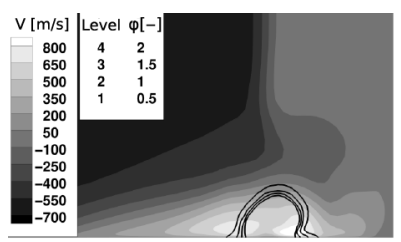

(d)

Core Inj. $J=1$

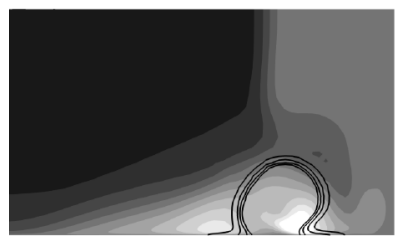

Core Inj. $J=3$ (b)

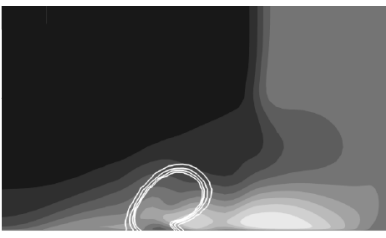

Intermediate Inj. $J=1$

(e)

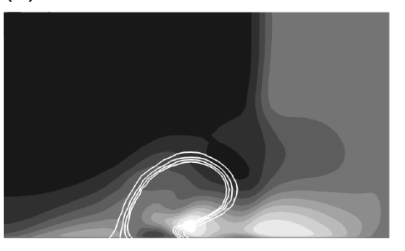

Intermediate Inj. $J=3$ (c)

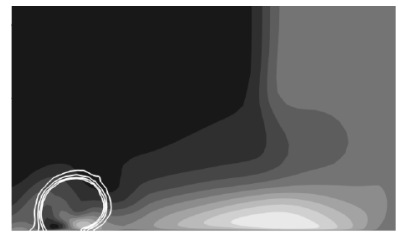

Separation Inj. $J=1$

(f)

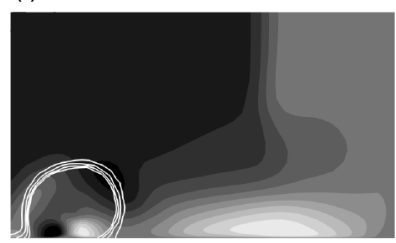

Separation Inj. $J=3$

Figure 9. Contours of spanwise velocity and lines of equivalence ratio $(\mathrm{Fr})$ on slices normal to the fin at $X_{i n j}=10 \mathrm{~mm}$. (Positive velocities are from right to left).

plume immediately after injection, making the plume span across a region of high velocity gradient. This produces an intense elongation of the plume shape starting immediately after injection. In the Separation injection case, the injector is located further from the high crosswise velocity region. As the vortex and plume grow, these interact similarly to the Intermediate injection case. Nonetheless, the increased distance between the jet and the high crossflow region produces a delay in the elongation compared with the Intermediate location case. For the Core injection case, the bulk of the plume is kept within a region of strong crossflow velocity, but with little velocity gradient across the plume in the direction normal to the flat plate. This reduces the level of elongation of the plume compared to the other two injector locations.

To quantify this effect and the evolution of the fuel plume shape as it moves downstream, the plume shape is analysed on multiple axial planes. For this, the plume edge is defined as the region enclosed by an iso-line of equivalence ratio $\phi=1$. Figure 10 compares the nondimensionalised perimeter to area ratio $D$ for all the test cases in this study. Note a circle is the geometric shape with the smallest possible value of $L / A$. Therefore, closed perimeter lengths should always return a value of $D>1$. However, the part of the perimeter adjacent to the flat plate surface is not effective for mixing (no mixing can occur over this length). After removing this section of the perimeter, values less than one $(D<1)$ are possible. Also, note the curves are only plotted up to the region where the fuel plume splits in two or more regions.

On each plot, the evolution of the plume with no vortex interaction (flat plate injection, FP.i.) is included for comparison. For flat plate injection with both $J=1$ and $J=3$ the $D$ values rapidly decrease and plateau as the plume reaches a constant quasi semi-circular shape (see Fig. 6(b)). On the contrary, in the cases where the plume interacts with the vortex, the perimeter to area ratio rises with increasing axial distance.

For a given injector location, the values of the non-dimensional parameter $D$ increase with $\alpha_{f i n}$ angles due to the higher intensity of the vortex, which induces a higher crossflow velocity. This produces a stronger crosswise elongation of the plume, increasing the nondimensionalized perimeter to area ratio. Comparing different injector locations, the Core 
(a)

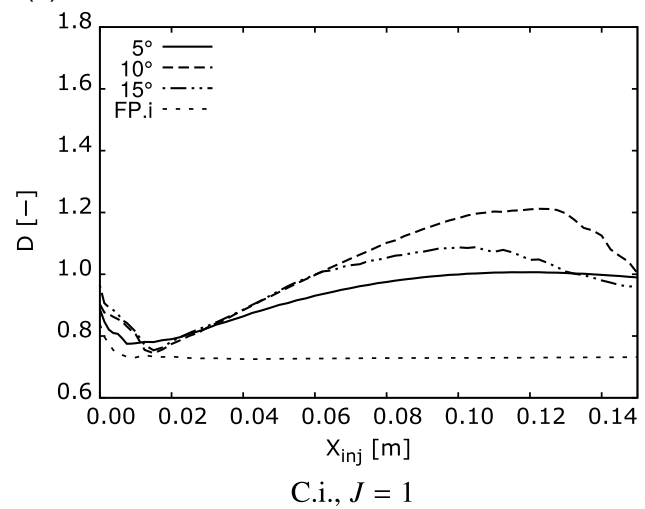

(c)

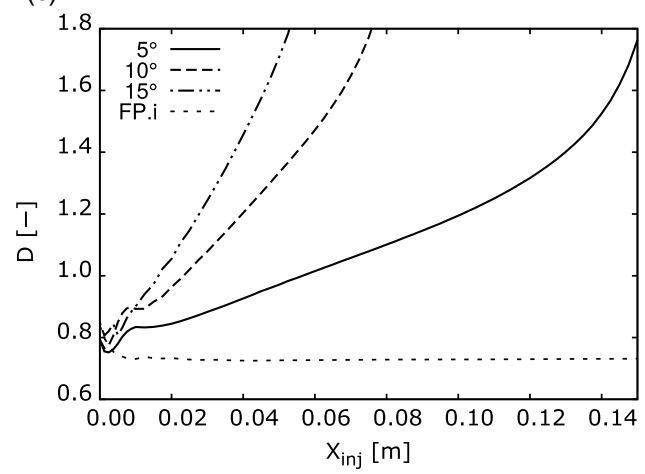

(e)

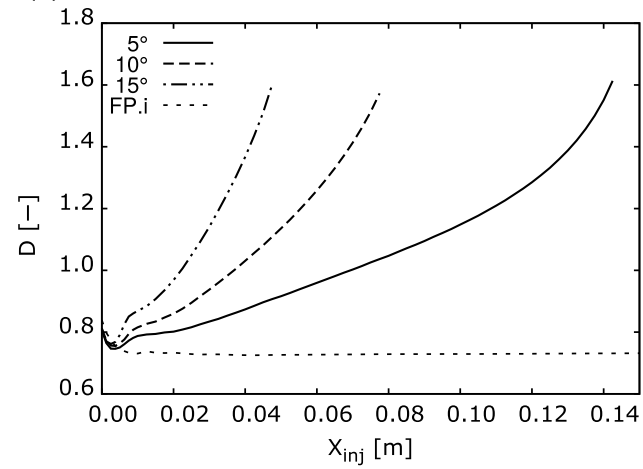

S.i., $J=1$ (b)

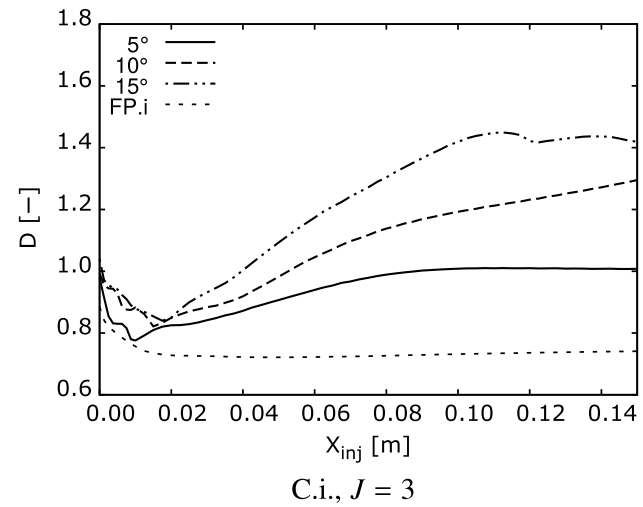

(d)

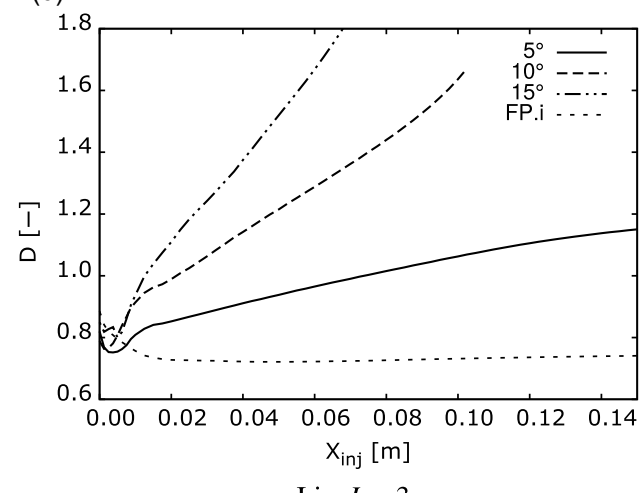

(f)

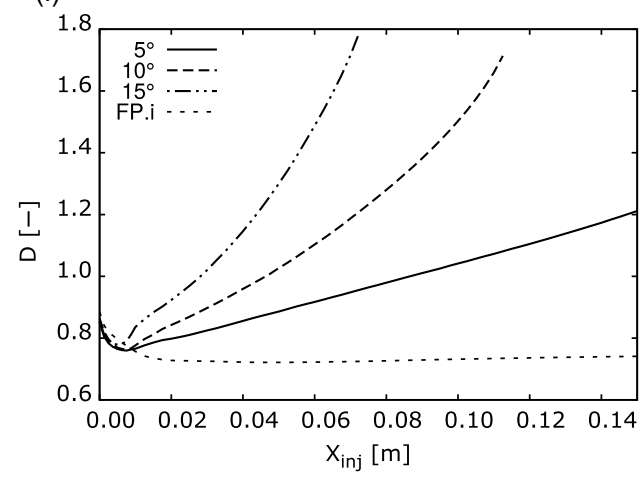

S.i., $J=3$

Figure 10. Non-dimensionalised perimeter-to-area ratio $D$.

injection plume is limited to significantly lower values of $D$ than the Intermediate and Separation injection plumes. This is due to the reduced velocity gradient across the plume as observed in Fig. 9. The Intermediate and Separation injection cases show very similar results, with slightly higher values in the Intermediate case. Comparing the Intermediate and Separation cases in Fig. 10, it can be observed that most of the difference in elongation occurs 
shortly downstream of the injector. As previously stated, the Intermediate location delivers the fuel into an optimal region, as it interacts with the vortex crossflow just after injection. The Separation injection case, however, takes longer to fully interact with the high velocity crossflow, producing a delay and slower stretching of the plume near the injector.

\subsection{Differences with the streamtube study}

The fuel plume generated at the presented flow conditions for the flat plate (no fin) injection case has an approximate diameter of $10 \mathrm{~mm}$ in the $J=3$ case, and $7 \mathrm{~mm}$ in the $J=1$ case. Moreover, this fuel plume is attached to the flat plate, as can be seen in Fig. 6(b). Therefore, the most similar study case using the streamtubes (or zero-mass particles circles) is the $\varnothing_{c}=10 \mathrm{~mm}$ case. The results of this case are shown in Fig. 7. From this figure, in the $\alpha_{f i n}=10^{\circ}$ case the injector location for maximum plume elongation sits between the Core and Intermediate injector locations. However, the injection case show a clear advantage of the Intermediate location. This discrepancy is due to the momentum of the injected hydrogen. The fuel is injected through an injector tilted at $45^{\circ}$ in the axial direction. The resulting axial velocity component causes the plume to move in the positive $X$ direction immediately downstream of the injector. In the conical coordinates system, this displaces the bulk of the plume towards lower $\mathrm{X} / \mathrm{Y}$ values (closer to the fin), moving the effective location of the injectors as seen in Fig. 7 to the left. This moves the effective injector location for the Intermediate and Separation injector to potentially higher $D$ values, and the Core injection location to potentially lower $D$ values.

Moreover, disturbance to the vortex flowfield caused by the injection process is also neglected in the zero-mass circle analysis. This has an important effect, altering the vortex crossflow velocity contours, which are the main driver of fuel plume elongation. This is specially visible in the Core injection case in Figs 9(a) and 9(d), in which the region of high crosswise velocity is thickened by injection. This causes an interesting difference between the Core injection cases, and the other two injection locations: the opposite trend of the parameter $D$ with increasing injection pressure. In the Core injection cases, $D$ values are higher for the high injection pressure cases. On the contrary, in the Intermediate and Separation injection cases, the opposite can be observed. Due to the thickening of the high crosswise velocity region, the Core injection case requires a higher injection momentum to penetrate further and produce a fuel plume spanning across the vortex velocity field. At low injection momentum, the Core injection case produces a plume that stays within a relatively constant spanwise velocity, limiting plume shape stretching. On the contrary, increasing the injectionto-freestream momentum flux ratio in the Intermediate and Separation cases places the bulk of the fuel plume further from the flat plate. In this cases, having the bulk of the flow further from the flat plate leaves a smaller portion of the plume to interact with the high crossflow velocity region near the flat plate, producing a drop in plume elongation.

\subsection{Mixing efficiency results}

To evaluate the effect of the vortex-injection interaction on mixing, the evolution of mixing efficiency $\left(\eta_{\text {mix }}\right)$ is analysed for each test case. For this, the flow is interrogated in a number of planes normal to the axial direction. On each plane, the mixing efficiency is calculated using Equation $2^{(33)}$, where $\rho, u$, and $c_{H 2}^{\text {stoic }}$ are the density, axial velocity, and stoichiometric $\mathrm{H}_{2}$ mass fraction $(0.0234)$. This provides the mixing efficiency value as a function of the axial distance 
(a)

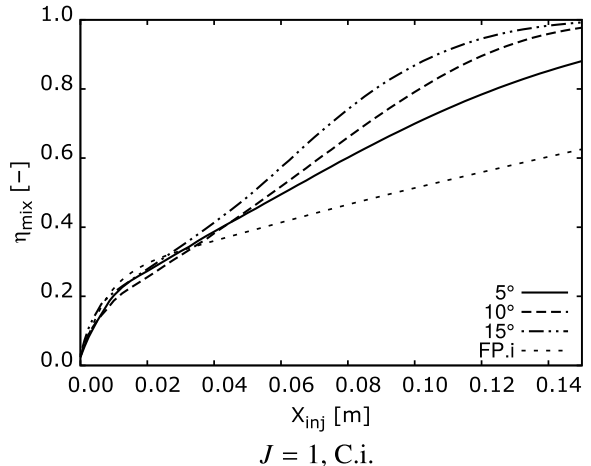

(c)

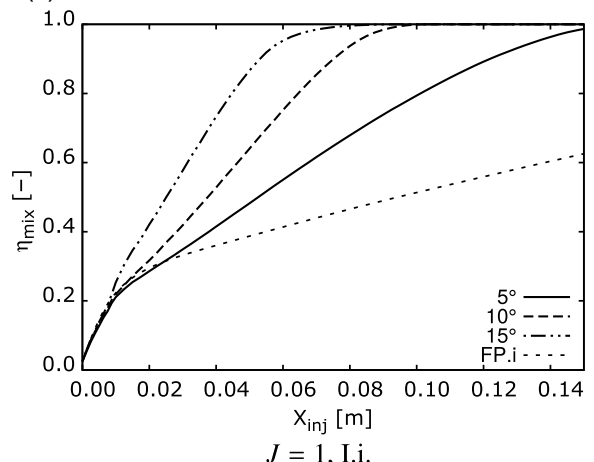

(e)

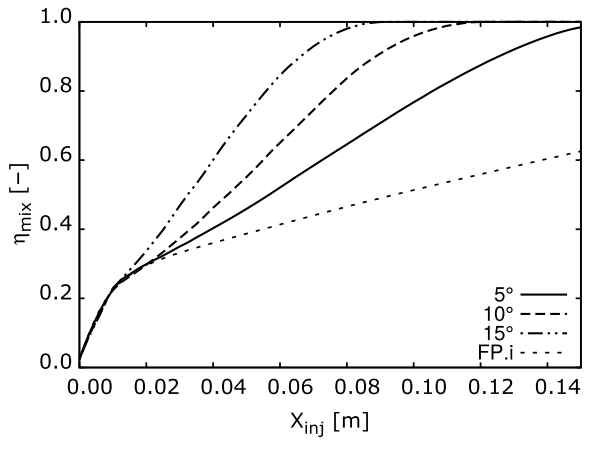

(b)

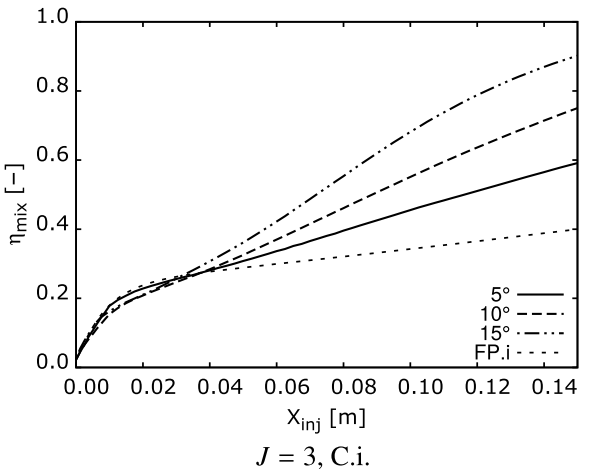

(d)

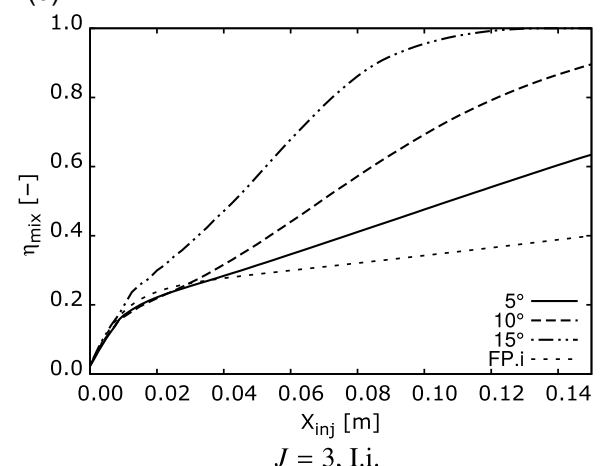

(f)

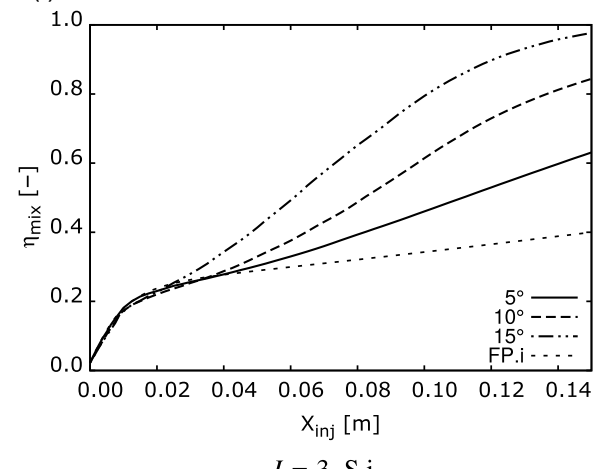

Figure 11. Mixing efficiency in streamwise direction for various injector locations and momentum ratios.

from the injector, $X_{i n j}$. The values obtained for all configurations are plotted in Fig. 11. In addition, the flat plate injection case is presented as a reference.

$$
\eta_{\text {mix }}=\frac{\iint c_{H 2}^{r} \cdot \rho \cdot u \cdot d y d z}{\iint c_{H 2} \cdot \rho \cdot u \cdot d y d z} \quad \text { where } \quad c_{H 2}^{r}= \begin{cases}c_{H 2} & c_{H 2} \leq c_{H 2}^{\text {stoic }} \\ \frac{1-c_{H 2}}{1-c_{H 2}^{\text {stoic }}} & c_{H 2}>c_{H 2}^{\text {stoic }}\end{cases}
$$

Figure 11 shows the impact of the vortex interaction on mixing rate. Mixing efficiency is highly increased by the vortex interaction. Higher fin angles, which produce stronger vortices, 
increase mixing rate, reducing distance to $\eta_{\text {mix }}=100 \%$. Comparison between the cases with different injection momentum shows that the $J=3$ cases have lower mixing efficiency along the whole mixing region. Comparing the different injector locations, the Intermediate location shows the highest performance for all fin angles and injection momentum ratios. The Separation injection location cases come second, with the Core injection cases showing the lowest values. This is coincident with the trends observed for the effective mixing area, indicating the relevance of this parameter in mixing rate. The Intermediate injection cases can have local mixing efficiencies up to 1.8 times higher than in the Core injection case and 1.2 times higher than the Separation injection case. Moreover the distance to fully mixing is reduced substantially in the Intermediate injection cases. These results show the strong link between effective mixing surface and mixing efficiency, as well as the potential gains in mixing rate achievable by appropriately injecting within the naturally formed streamwise vortices in scramjets.

It is worth noting that the averaged nature of the RANS solution eliminates the effect of vortex instabilities and vortex meandering, which may create a fluctuating relative location between vortex Core and injector. These could be detrimental in that they limit the time this relative location is optimal, or beneficial in that the vortex fluctuation could disperse the fuel more effectively across the combustor. Different fuels could also have a slightly different behaviour when interacting with the incoming vortex, as different relative densities would generate different baroclinic torque that would affect the fuel-vortex interaction and fuel transport.

\subsection{Mixing efficiency and plume shape}

To highlight the correlation between non-dimensionalised fuel plume perimeter-to-area ratio, $D$ and improvement in mixing efficiency, Figs 12 and 13 show both quantities normalized by the corresponding Core injection case data.

These two quantities can only be compared close to the injector, as mixing efficiency asymptotes to one as fully mixed conditions are approached. Focusing on the region between $X_{i n j}=0$ to $X_{i n j}=0.06$, the normalized values of mixing efficiency in Fig. 12 rise very rapidly just downstream of the injector, and then continue growing with a lower gradient. For the $J=1$ cases, the normalized Intermediate injection cases (I.i./C.i.) rapidly reach about 1.4 , 1.2 and 1.0 in the $15^{\circ}, 10^{\circ}$ and $5^{\circ}$ fin angles respectively. The rapid increase in normalised D parameter for the same cases in Fig. 13 reach very similar values. Moreover, the subsequent slower rise in normalized mixing efficiency and D parameter up to $X_{i n j}=0.06$ also follows very similar trends for both parameters before normalized mixing efficiency curves start asymptotically going to one. This similarity is also visible for the Separation injection cases as well as the $J=3$ cases comparing Figs 12 and 13. This similar behavior for both parameters: mixing efficiency and D parameter, strengthens the link between plume shape and mixing efficiency rate, showing the relevance of the fuel plume shape for mixing enhancement.

\subsection{Losses and Entropy generation}

Generation of losses is another relevant parameter when selecting the best fueling strategy for overall performance. To quantify this effect in the vortex-injection interaction the entropy in the flowfield is calculated at each cell using equation 3 , where the reference values $P_{\infty}$ and $\rho_{\infty}$ are the freestream inflow conditions.

$$
S=c_{v} \ln \left(\frac{P}{P_{\infty}}\right)+c_{p} \ln \left(\frac{\rho_{\infty}}{\rho}\right)
$$



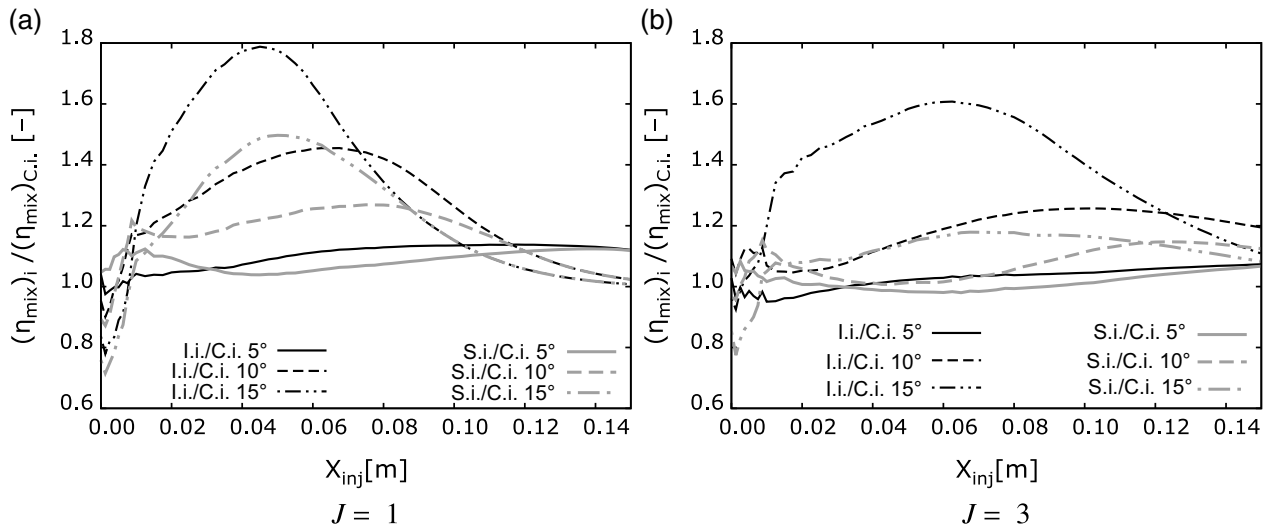

Figure 12. Mixing efficiency normalized by the corresponding Core injection case.

(a)

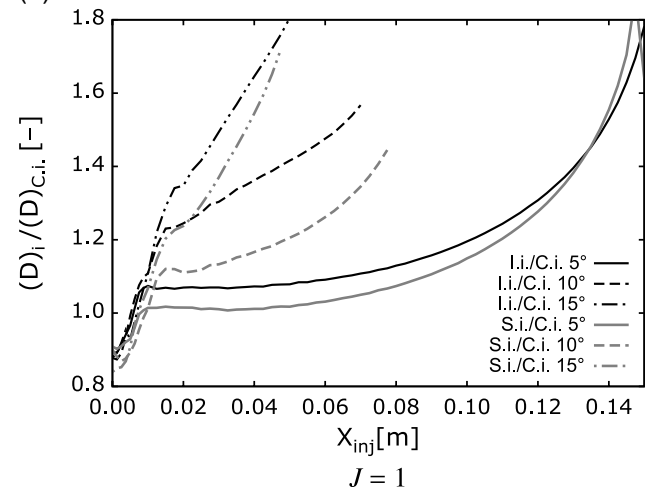

(b)

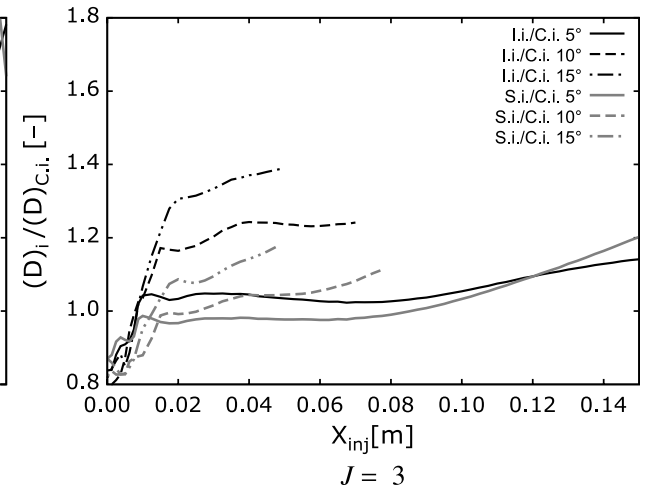

Figure 13. Non-dimensionalised perimeter-to-area ratio $D$ normalized by the corresponding Core injection case.

Figure 14 shows the evolution of entropy from the fin leading edge to the end of the domain evaluated at multiple axial planes. Entropy shows a steady increase from the fin leading edge $\left(X_{i n j}=-0.10\right)$ due to the presence of the fin shock wave. Moreover, this shock produces higher entropy for higher fin angles. In the vicinity of the injector, a steep rise in entropy is produced due to the injection process. For all fin angle and injection momentum ratio cases the Intermediate injection location generates the most severe increase in entropy in the injector vicinity, followed by the Separation injector location. However, the entropy values tend to reach very similar levels shortly downstream of the injector. Although the Core injector generated the lowest increase in entropy in the injector vicinity, further downstream entropy levels for this injector location tend to surpass the other injector locations. This is specially visible in the $J=3$ cases in Fig. 14(b).

The vortex-injection cases and the case without vortex (flat plate injection, FP.i.) cannot be compared directly due to the lack of a fin shock in the later. However, to compare the effect on entropy of injecting within the vortex, the entropy rise $(\Delta S)$ from the injector location is presented if Fig 15 for the $\alpha=5^{\circ}$ cases and the flat plate injection case with $J=1$. This figure shows that the initial rise in entropy due to the injection process is very similar for 
(a)

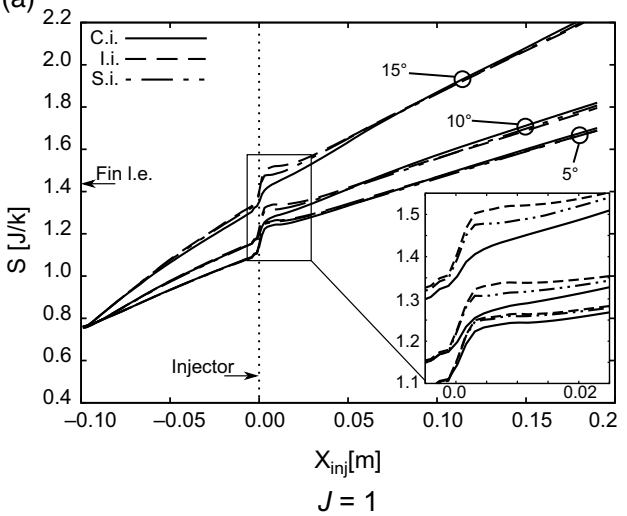

(b)

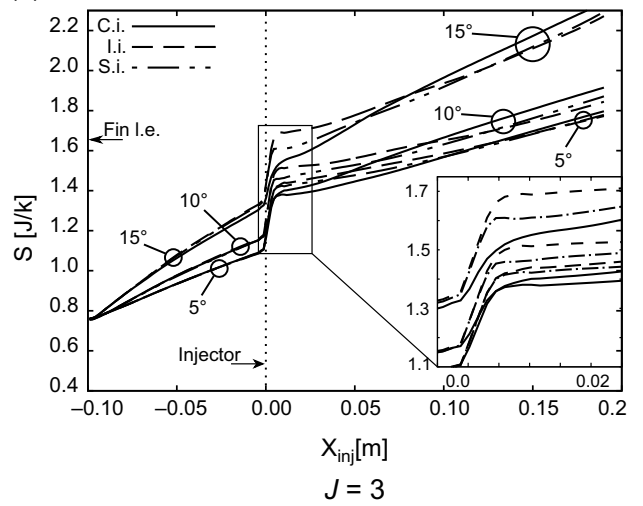

Figure 14. Entropy evolution from fin leading edge to end of domain.

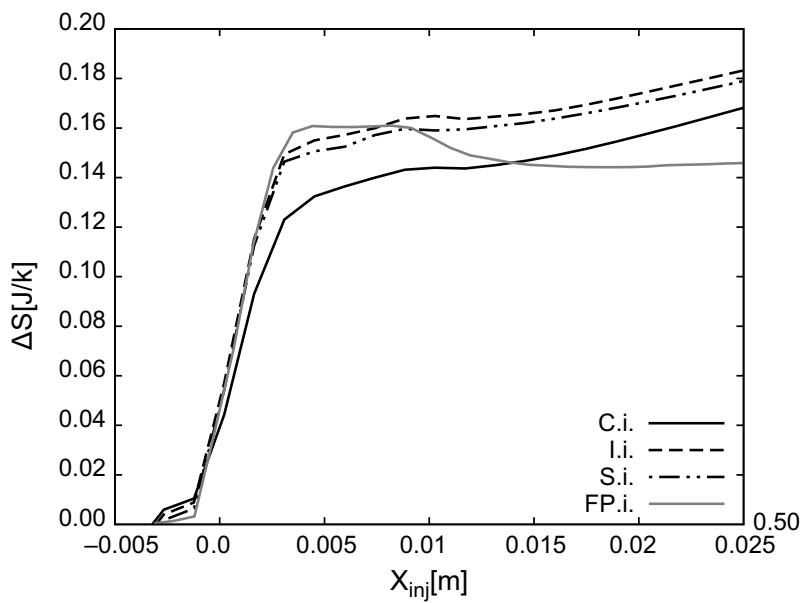

Figure 15. Entropy rise at injector location for $\alpha=5^{\circ}$ and FP.i. $J=1$ cases.

all presented cases. Interestingly, a slightly higher entropy rise in the flat plate injection case exists. In this case injection takes place into the undisturbed freestream, with a higher Mach number. Hence the loses in the injection bow shock are increased. As the injector is moved towards the vortex core, the loses are slightly reduced thanks to the reduced Mach number within the vortex. The same is true for the $J=3$ cases. This result further supports the fact that utilising existing vortices produces none or negligible increase in loses. Indeed, in this case entropy related to the injection process is slightly reduced.

Shortly downstream of the injector, the flat plate injection case curve diverges from the others due to the lack of a fin shock in this case. The region of entropy decrease in the FP.i. case is due to the high temperature of the gas near the flat plate wall caused by the injection bow shock, which produces a high amount of heat lost to the constant temperature wall.

Due to the minimal differences in entropy generation for the different injector locations, especially as the values reach almost equal levels a short distance downstream of the injector, there is no preference fora a particular injector location with respect to loss generation. 
Moreover the entropy generated by injecting within the vortex is slightly lower than the baseline case of injecting in the freestream, as shown in Fig. 15. This confirms that for the conditions investigated, by injecting into the vortex, better mixing is attained without causing increased losses.

\subsection{CONCLUSIONS}

A simplified geometry generating vortices equivalent to those found in scramjet inlets was used to study the effect of streamwise vortices on porthole injected fuel plume shape and mixing rate. Three different vortex intensities, three injector locations, and two injection-tofreestream momentum flux ratios were analysed. To evaluate the quality of a fuel plume shape for mixing purposes parameter $D$, a non-dimensional measure of plane perimeter to plume cross-sectional area, is proposed. A study to assess the potential of the vortex flowfield to produce fuel plume elongation was performed using rings of zero-mass particles. The results identify the regions of the vortex flowfield with highest potential for fuel plume elongation for different vortex intensities and fuel plume sizes and locations. Moreover, it is shown that the region adjacent to the flat plate is the largest contributor to elongation. The actual fuel-vortex interaction was studied by injecting $H_{2}$ fuel at three different spanwise locations within the vortex. The Intermediate injector location produced the highest plume elongation followed by the Separation injector, while the Core injector clearly underperformed compared to the former. For the Intermediate injector case a maximum increase of up to 2.4 times in effective area for mixing, compared to the case with no vortex interaction is achieved. Mixing efficiency shows very similar trends to the $D$ parameter curves, highlighting the importance of fuel plume elongation on mixing rate. Very substantial gains in mixing rate are obtained thanks to the interaction between vortex and fuel jet. This substantially reduces the distance between injection and location where $100 \%$ mixing efficiency is obtained. This improvement is most significant for the Intermediate injection location. Moreover, the consistently higher improvement on fuel mixing rate using the Intermediate injector location suggests that this is the case for a wide range of vortex intensities, ranging from the weak naturally occurring vortices in a shape-transition inlet to the strong vortices formed in typical 2D scramjet inlets. Analysis of the entropy generation both in the injector proximity and far downstream shows that injection strategy does not create losses higher than a flat plate injection. Therefore, this work shows the possibility of substantially improving mixing rate while incurring no increase in losses by using the appropriate injection strategy that exploits the presence of scramjet inlet generated vortices. These features of mixing enhancement without losses or increased heat loads are critical for high Mach number access to space Scramjets.

\section{ACKNOWLEDGEMENTS}

This research was supported under Australian Research Council's Discovery Projects funding scheme (Project DP130102617-The science of scramjet propulsion). This research was undertaken with the assistance of resources from the National Computational Infrastructure (NCI), which is supported by the Australian Government, and by resources provided by The Pawsey Supercomputing Centre with funding from the Australian Government and the Government of Western Australia. The author would also like to thank the School of Mechanical and Mining Engineering at UQ for the financial support. 


\section{REFERENCES}

1. Smart, M.K. and TetLow, M.R . Orbital delivery of small payloads using hypersonic airbreathing propulsion, $J$ Spacecraft Rockets, 2009, 46, (1), pp 117-125. https://doi.org/10.2514/1.38784

2. СоOK, $\mathrm{S}$ and HueTER, $\mathrm{U}$. NASA's integrated space transportation plan 3rd generation reusable launch vehicle technology update, Acta Astronaut, 2003, 53, (4-10), pp 719-728. https://doi.org/ 10.1016/S0094-5765(03)00113-9

3. Marshall, L.A., Bahm, C., Corpening, G.P. and Sherrill, R . Overview with Results and Lessons Learned of the X-43A Mach 10 Flight. AIAA/CIRA 13th International Space Planes and Hypersonics Systems and Technologies Conference, Capua, Italy, 2005. https://doi.org/ $10.2514 / 6.2005-3336$

4. McClinton, C.R . X-43 - Scramjet Power Breaks the Hypersonic Barrier: Dryden Lectureship in Research for 2006, 44th AIAA Aerospace Sciences Meeting and Exhibit, Aerospace Sciences Meetings, Reno, Nevada, 2006. https://doi.org/10.2514/6.2006-1

5. Hank, J.M., Murphy, J.S. and Mutzman, R.C . The X-51A Scramjet Engine Flight Demonstration Program, 15th AIAA International Space Planes and Hypersonic Systems and Technologies Conference, Dayton, Ohio, 2008. https://doi.org/10.2514/6.2008-2540

6. Vishwakarma, M. and Vaidyanathan, A . Experimental study of mixing enhancement using pylon in supersonic flow, Acta Astronaut, 2016, 118, pp 21-32. https://doi.org/10.1016/ j.actaastro.2015.09.011

7. Aguilera, C. and Yu, K.H . Effect of Fin-Guided Fuel Injection on Dual Mode Scramjet Operation, Propulsion and Energy Forum. 50th AIAA/ASME/SAE/ASEE Joint Propulsion Conference, 2014, Cleveland, Ohio. https://doi.org/10.2514/1.B36305

8. Aravind, S. and Kumar, R. Supersonic combustion of hydrogen using an improved strut injection scheme, Int. J. Hydrogen Energy, 2019, 44, (12), pp 6257-6270. https://doi.org/10.1016/ j.ijhydene.2019.01.064

9. Fureby, C., Nordin-Bates, K., Petterson, K., Bresson, A. and Sabelnikov, V.A . Computational study of supersonic combustion in strut injector and hypermixer flow fields. Proceedings of the Combustion Institute, 2015, 35, (2), pp 2127-2135. https://doi.org/10.1016/j.proci.2014.06.113

10. Li, L.-Q, Huang, W., Yan, L., Zhao, Z-T. and Liao, L . Mixing enhancement and penetration improvement induced by pulsed gaseous jet and a vortex generator in supersonic flows, Int. J. Hydrogen Energy, 2017, 42, (30), pp 19318-19330. https://doi.org/10.1016/j.ijhydene. 2017.06.014

11. Li, L.-Q, HuAnG, W. and Yan, L . Mixing augmentation induced by a vortex generator located upstream of the transverse gaseous jet in supersonic flows, Aerosp. Sci and Technol, 2017, 68, pp 77-89. https://doi.org/10.1016/j.ast.2017.05.016

12. Li, L.-Q., Huang, W., Fang, M., Shi, Y-L., Li, Z-H. and Peng, A.P . Investigation on three mixing enhancement strategies in transverse gaseous injection flow fields: A numerical study, Int. $J$ Heat and Mass Transfer, 2019, 132, pp 484-497. https://doi.org/10.1016/j.ijheatmasstransfer. 2018.12.038

13. Fuller, R.P., Wu, P.K., Nejad, A.S. and Schetz, J.A . Comparison of physical and aerodynamic rams as fuel injectors in supersonic flow. J Propul Power, 1998, 14, (2), pp 135-145. https://doi.org/10.2514/2.5278

14. Malo-Molina, F.J., Gaitonde, D.V., Ebrahini, H.B. and Ruffin, S.M . Three-dimensional analysis of a supersonic combustor coupled to innovative inward-turning inlets, AIAA J, 2010, 48, (3), pp 572-582. https://doi.org/10.2514/1.43646

15. James, E. Barth, J.E., Wise, D.J., Wheatley, V. and Smart, M.K. Tailored Fuel Injection for Performance Enhancement in a Mach 12 Scramjet Engine, International Space Planes and Hypersonic Systems and Technologies Conferences, 2015, Glasgow, Scotland. https://doi.org/ $10.2514 / 6.2015-3614$

16. Gardner, A., Paull, A. and McIntyre, T. Upstream porthole injection in a 2-D scramjet model. Shock Waves, 2002, 11, (5), pp 369-375. https://doi.org/10.1007/s001930200120

17. Alvi, F.S. and Settles, G.S. Physical model of the swept shock wave/boundarylayer interaction flowfield, AIAA J, 1992, 30, (9), pp 2252-2258. https://doi.org/10.2514/3.11212

18. Smart, M.K . Design of three-dimensional hypersonic inlets with rectangular-toelliptical shape transition, J Propul Power, 1999, 15, (3), pp 408-416. https://doi.org/10.2514/2.5459 
19. Smart, M.K. and TreXler, C.A. Mach 4 performance of hypersonic inlet with rectangular-toelliptical shape transition, J Propul Power, 2014, 20, (2), pp 288-293. https://doi.org/10.2514/ 1.1296

20. Barth, J.E., Wheatley, V., Smart, M.K., Petty, D.J. and Basore, K.D. Flow physics inside a shape-transitioning scramjet engine, 18th AIAA/3AF ISPHSTC, 24-28 September, Tours, France 2012. https://doi.org/10.2514/6.2012-5888

21. Llobet, J.R., JaHN, I.H. and Gollan, R.J. Effect of Streamwise Vortices on Scramjets Porthole Injection Mixing, Proceedings for the 20th AIAA International Space Planes and Hypersonic Systems and Technologies Conference, Glasgow, Scotland, 2015. https://doi.org/10.2514/6.20153597

22. Kubota, H. and Stollery, J.L. An experimental study of the interaction between a glancing shock wave and a turbulent boundary layer, J Fluid Mech, 1982, 116, pp 431-458. https://doi.org/10.1017/S0022112082000548

23. Llobet, J.R., Barth, J.E. and JahN, I.H. , Vortex Tracking Algorithm for Hypersonic Flow in Scramjets, 19th AFMC, 8-11 December, Melbourne, Australia, 2014.

24. Llobet, J.R., JAHN, I.H. and Gollan, R.J. Effect of vortex-injection interaction on wall heat transfer in a flat plate and fin corner geometry, Asia-Pacific International Symposium on Aerospace Technology, Toyama, Japan, 2016. https://doi.org/10.2322/tastj.15.a17

25. Hunt, J.C.R., WRAY, A.A. and Moin, P. Eddies, streams, and convergence zones in turbulent flows, Center for Turbulence Research, Proceedings of the Summer Program, 1988, N89-24555, pp 193-208.

26. McGilvray, M. Scramjet testing at high enthalpies in expansion tube facilities, $\mathrm{PhD}$ thesis, The University of Queensland, 2008.

27. SANChO, J. Scramjet testing at high total pressure, $\mathrm{PhD}$ thesis, The University of Queensland, 2015.

28. Nompelis, I., Drayna, T.W. and Candler, G.V. Development of a Hybrid Unstructured Implicit Solver for the Simulation of Reacting Flows Over Complex Geometries, 34th AIAA Fluid Dynamics Conference and Exhibit, Portland, Oregon, 2004. https://doi.org/10.2514/6.2004-2227

29. Wright, M.J., Candler, G.V. and Bose, D. Data-parallel line relaxation method for the navierstokes equations, AIAA J, 1998, 36, (9), pp 1603-1609. https://doi.org/10.2514/2.586

30. Roache, P.J. Perspective: A Method for Uniform Reporting of Grid Refinement Studies. ASME J Fluids Eng, 1994, 116, (3), pp 405-413. https://doi.org/10.1115/1.2910291

31. Llobet, J.R., BASORE, K.D., Gollan, R.J. and JAHN, I.H. Experimental and Numerical Heat Transfer from Vortex-Injection Interaction in Scramjet Flowfields. (Under review.)

32. Peterson, D.M., Boyce, R.R. and WheatLey, V. Simulations of mixing in an inlet-fueled axixymmetric scramjet, AIAA J, 2013, 51, (3), pp 2823-2832. https://doi.org/10.2514/1.J052480

33. LeE, S.H. Characteristics of dual transverse injection in scramjet combustor, part 1: Mixing. $J$ Propul Power, 2006, 22, (5), pp 1012-1019. https://doi.org/10.2514/1.14180 